\title{
Programa de Ergonomía Participativa para la Prevención de Trastornos Musculoesqueléticos. Aplicación en una Empresa del Sector Industrial
}

\author{
PARTICIPATIVE ERGONOMIC PROGRAM FOR PREVENTION OF MUSCULOSKELETAL DISORDERS APPLICATION \\ IN A COMPANY OF INDUSTRIAL SECTOR
}

\author{
Rodrigo Pinto Retamal ${ }^{1}$ \\ 1. Especialista Ing. Ocupacional.
}

\section{RESUMEN}

Se propone como estrategia para el control de los Trastornos Musculoequeléticos (TME) un Programa de Ergonomía Participativa (PEP). La aplicación de este programa se divide en 5 etapas: Análisis de empresa, evaluación de riesgos ergonómicos, construcción del plan de acción, ejecución del plan de acción y verificación de medidas de control.

La base que sustenta al PEP es la conformación de un Grupo de Ergonomía en la empresa (Grupo Ergo), quien estará a cargo de la identificación, evaluación e implementación de medidas de control con la asesoría de un profesional de Ergonomía.

Las metodologías de evaluación de riesgos a utilizar por el Grupo Ergo serán las indicadas en la Normativa Chilena (Ley 20.001 DS Nº3/2005 y Decreto $\mathrm{N}^{\circ} 4$ que modifica el DS $\mathrm{N}^{\circ} 594$ ) que exigen la aplicación de las herramientas: Manual Asessments Charts (MAC) para identificar y evaluar tareas de Manejo Manual de Carga en Levantamiento-descenso, transporte, levantamiento, descenso en equipo y las tablas de Liberty Mutual para tareas de empuje y arrastre de carga; y, por otra parte, la Norma Técnica de Trastornos Musculoequeléticos de Extremidad Superior (MINSAL, 2011) establece la aplicación de una Lista de chequeo contenida en la Norma ISO 11228-3 para la identificación y evaluación de tareas que involucren movimientos repetitivos, postura, fuerza, tiempos de recuperación y factores adicionales, respectivamente.

Se presenta la aplicación del PEP en una empresa del rubro industrial chileno, planteándose como objetivo cumplir con las etapas del programa en un período de 4 meses. Se establece como objetivo del PEP lograr implementar un 70\% de las medidas consideradas denivel "simples". Los resultados reflejaron el cumplimiento de las etapas, logrando implementar un 79\% de las medidas simples y un 27\% de las medidas complejas. Pese a que es una experiencia primaria en Chile, se pudieron observar beneficios indirectos en la ejecución del PEP, tales como generar una cultura en la empresa respecto a temas de Ergonomía, lograr el compromiso de los ejecutivos en la implementación de las medidas de control y el cumplimiento de legislación chilena respecto a Ergonomía.

(Pinto R, 2015. Programa de Ergonomía Participativa para la Prevención de Trastornos Musculoesqueléticos. Aplicación en una Empresa del Sector Industrial. Cienc Trab. May-Ago; 17 [53]: 128-136).

Palabras clave: ERGONOMIA PARTICIPATIVA, TRASTORNOS MUSCULOESQUELÉTICOS, LEY 20.001, NORMA TÉCNICA TMERT MINSAL.

\section{ABSTRACT}

It is proposed as a strategy to control Musculoskeletal disorders (MSDs) Participatory Ergonomics Program (PEP). The implementation of this program is divided into 5 stages: Company Analysis, ergonomic risk assessment, construction of the action plan, implementation of the action plan and verification of control measures.

The base that sustains the PEP is the creation of a Group of Ergonomics in the company (Ergo Group), who will be responsible for the identification, evaluation and implementation of control measures with the assistance of a professional ergonomics.

The risk assessment methodologies to be used by Ergo Group shall be indicated in Chilean law (Law 20,001 DS No. 63/2005 and Decree No. 4 amending DS No. 594) that requires the application of tools: Manual assessments charts (MAC) to identify and evaluate tasks Burden Handling Manual lifting-lowering, transport, lifting, lowering team and Liberty Mutual tables to pushing and load dragging tasks, and, moreover, the Technical Regulations Musculoskeletal disorders Upper Extremity (MINSAL, 2011) establishes the application of a checklist contained in ISO 11228-3 for the identification and evaluation of tasks that include repetitive movements, posture, strength, recovery times and additional factors, respectively.

The implementation of PEP is presented in a company of the Chilean industrial sector, setting out as objective to comply with the program steps over a period of 4 months. It sets the objective of achieving PEP implement 70\% of the measures consider of "simple" level.

The results showed compliance with stages, achieving 79\% implement simple measures and $27 \%$ of complex measures. Although it is a primary experience in Chile, indirect benefits could be observed in the implementation of PEP, such as a culture in the company on issues of ergonomics, to achieve the commitment of the executive in the implementation of control measures and compliance with Chilean legislation on Ergonomics.

Keywords: PARTICIPATORY ERGONOMICS, MUSCULOSKELETAL DISORDERS, LAW 20,001, TECHNICAL STANDARD TMERT MINSAL.
Correspondencia / Correspondence:

Rodrigo Pinto Retamal.

Ramón Carnicer 163. Santiago de Chile.

Tel. 02-25157555

e-mail: rpinto@achs.cl

Recibido: 07 de Febrero de 2015 / Aceptado: 23 de Abril de 2015

\section{INTRODUCCION}

En Chile la Ergonomía como disciplina ha tenido un desarrollo de más de 40 años, durante los cuales sólo en la última década se han desarrollado Normativas que obligan a las empresas a gestionar sus riesgos ergonómicos de naturaleza biomecánica, fundamentalmente lo que refiere al control de los riesgos por Manipulación de 
Cargas y tareas repetitivas que afecten las extremidades superiores. Bajo este marco legal las mutuales de empleadores (Ley 16.744) han debido desarrollar estrategias que permitan asesorar a las empresas en la gestión de estos riesgos. En términos generales, la asesoría estándar que brindan las mutuales se traduce en la visita de un Experto en Prevención de riesgos o bien de un profesional con formación en Ergonomía, quien realiza una o más visitas para luego generar un diagnóstico y finalmente entregar un informe con recomendaciones a la empresa respecto al problema en cuestión.

Según la experiencia recopilada, estos informes resultan difíciles de interpretar por parte de los técnicos de las empresa y la implementación de las medidas recomendadas es bajo respecto al número de asesorías realizadas (datos del Instituto de Salud Pública de Chile (ISP) reflejan esta situación); a modo de ejemplo y que a pesar de que los TME en Chile son de alta prevalencia, solo un 21,5\% de los empleadores y un 18,4\% de los trabajadores respectivamente, refieren estar trabajando en medidas preventivas en este ámbito. En el año 2011, las acciones dirigidas a prevenir trastornos de tipo músculo-esqueléticos en las empresas, según declaran los mismos empleadores, solo alcanza a un $21,7 \%{ }^{1}$

Algunas de las posibles causas que explican este fenómeno podrian ser: la falta de cultura preventiva en las empresas, falta de conocimientos de Ergonomía, escaso involucramiento por parte de los ejecutivos de las empresas en la solución de estos problemas, falta de estrategia de seguimiento por parte de los Ergónomos que realizan estas asesorías, entre otras.

Una alternativa para revertir lo anterior, es utilizar una estrategia en la cual se involucren técnicos y trabajadores de la empresa en la identificación, evaluación y control de sus riesgos con la asesoría de un profesional de Ergonomía competente en la materia. La Ergonomía participativa como estrategia de empoderamiento de los trabajadores aparece como una alternativa a utilizar en las empresas en Chile. Países como Canadá, Inglaterra y España, de manera incipiente, han propuesto modelos basándose en este concepto con buenos resultados. ${ }^{2}$

\section{Antecedentes sobre TME a nivel nacional}

A nivel nacional, sin bien las estadísticas no indican la magnitud real del problema dado el sub-diagnóstico y escasa homogeneidad en el registro de información, existen datos de la Asociación Chilena de Seguridad que nos señalan que las Enfermedades Profesionales músculo-esqueléticas constituyen un 30\% del total de Enfermedades profesionales. ${ }^{3}$

Luego, al hacer un análisis del comportamiento de Enfermedades profesionales por sector económico, según estadísticas de la ACHS en un período de tiempo comprendido entre los años 2009-2012, el sector que presenta un mayor número de casos de enfermedades profesionales musculoesqueléticas de extremidad superior respecto a su masa, es el sector pesquero con una tasa de 18,1 seguido del sector Forestal maderero 5,2 y Gubernamental-Municipalidades 5,0 (Tasa en función de número de casos por cada 1000 trabajadores. Fuente: Base de datos Gerencia de Prevención ACHS).

Respecto al Síndrome de Dolor Lumbar, este diagnóstico se ubica como la tercera patología en relación al número de casos y días de tratamiento en el periodo 2001-2012, esto para una muestra de 2.000.000 de trabajadores y 40.000 empresas. ${ }^{4}$ Luego, si analizamos el número de casos reportados durante el año 2011 por sector económico, vemos que el sector construcción es el que tiene un mayor impacto con una tasa de 15,4, seguido del sector Industrial $(6,7)$ y Forestal $(4,4)$.

\section{Ergonomía participativa}

Se define como: "Una Estrategia que involucra a las personas en la planificación y control de una parte significativa de su trabajo, con el suficiente conocimiento y capacidad para influir sobre los procesos y sus resultados con el objetivo de conseguir metas específicas, asociadas al control de problemas de Ergonomía" [Haines y Wilson, 1998]. ${ }^{5}$

Un elemento central de este enfoque es la constitución de un grupo de trabajo en la empresa denominado GRUPO ERGO, que incluye representantes de distintas áreas (Seguridad y Salud en el Trabajo, Recursos Humanos, Producción, Mantención, entre otros) $\mathrm{y}$ cuyas funciones, en términos amplios, será el diagnóstico y evaluación del riesgo, además del seguimiento de la implementación de las medidas propuestas y su evaluación. Por su parte, los técnicos en Prevención de Riesgos y Ergonomía aportan su conocimiento y experiencia en las fases centrales de desarrollo del proceso, especialmente en relación con la capacitación en el uso de herramientas de evaluación, las propuestas de mejora y el ensayo de las intervenciones.

En las dos últimas décadas el interés por el desarrollo de la Ergonomía Participativa ha aumentado paulatinamente en muchos países. Algunas revisiones de la literatura técnica internacional describen una tendencia creciente de publicaciones relacionadas a estas intervenciones en distintos sectores laborales. ${ }^{2,6}$ En Chile es aún una práctica poco conocida y menos aún aplicada.

En España, se han propuesto modelos adaptados de publicaciones de otros países que se encuentran bajo el contexto de su propia legislación. El método ERGOPAR ${ }^{7}$ señala que el implementar una intervención participativa contribuye al cumplimiento de la normativa en materia de prevención de riesgos laborales ya que:

- Promueve la seguridad y salud de los trabajadores.

- Se proponen intervenciones adaptadas a las circunstancias de la empresa.

- Se reduce la resistencia al cambio.

- Acorta los tiempos de implementación de cambios, dada la implicación de personas con capacidad de decisión y personas que conocen sus puestos de trabajo.

- Permite visualizar el compromiso de la empresa en prevención de riesgos laborales.

- Se mejoran las relaciones laborales, a través de la comunicación.

Instituciones como NIOSH de Estados Unidos, HSE de Inglaterra y la Agencia Europea para la Seguridad y Salud en el Trabajo, mencionan a la Ergonomía Participativa como estrategia eficaz para la prevención de los trastornos musculoesqueléticos de origen laboral..$^{5,8,9,10}$

Uno de los fundamentos principales de este enfoque es la participación activa de los trabajadores, sobre todo en el proceso de identificación de soluciones, lo que permite ocupar su conocimiento de las tareas y asegurar un mayor grado de aceptación de las soluciones propuestas.

\section{OBJETIVOS}

Según lo expuesto anteriormente, el presente proyecto tiene como objetivo principal proponer un programa participativo para asesorar a las empresas en la gestión de los riesgos asociados a la generación de TME y que se encuentran vinculados a la aplicación de las normativas nacionales. Esta propuesta se aplica en una empresa industrial y se analizan los resultados obtenidos. 
Los objetivos específicos son:

- Conformar un Grupo de Ergonomía (Grupo Ergo) en la empresa.

- Lograr que el Grupo Ergo evalúe tareas de manejo manual de carga utilizando metodología MAC (Ley 20.001 DS №63).

- Lograr que el Grupo Ergo evalúe tareas con riesgo de generar TME de extremidad superior utilizando Lista de Chequeo TMERT MINSAL.

- Definir un Plan de acción a desarrollar en la empresa con recomendaciones específicas a los problemas detectados.

- Implementar un 70\% de las medidas simples contenidas en el plan de acción.

- Cumplir con la legislación chilena vinculada con temas de Ergonomía.

\section{METOdOLOGÍA}

Propuesta de Programa de Ergonomía Participativa (PEP) EL PEP es una adaptación de las etapas del modelo de gestión de riesgos utilizado por la ACHS (Sistema Preventivo) para asesorar a las empresas en materia de prevención de riesgos laborales. El Sistema preventivo es una metodología de trabajo que comienza con el levantamiento de la situación de la empresa en torno a la cultura organizacional y preventiva, abordando las temáticas de seguridad y salud en el trabajo (SST), para definir una estrategia a seguir, lo que dependerá de las variables tales como: el interés de la empresa por los temas de SST, la existencia de sistemas de gestión en ella, la clasificación de la empresa según sus tasas de accidentalidad, fatalidad, siniestralidad, etc. Posteriormente, se identifican los peligros existentes, evaluando los riesgos asociados y estableciendo medidas de control para los niveles más críticos por medio de un equipo multidisciplinario y un trabajo conjunto entre ACHS y la empresa. Además de lo anterior, la metodología apunta a incorporar medidas de control de riesgos en los puestos de trabajo. ${ }^{11}$

El PEP, por lo tanto, adapta las 5 fases del Sistema Preventivo ACHS a la realidad de la empresa en temas de Ergonomía, especificamente en lo que refiere al control de los TME localizados en la espalda y extremidades superiores en los puestos de trabajo que presenten tareas críticas asociadas a la Manipulación de cargas y Trabajo repetitivo.

El líder del PEP es un Ergónomo que interactúa con un Grupo de Ergonomía (Grupo Ergo) conformado en la empresa. A diferencia de otro tipo de asesorías en las cuales el Ergónomo tiene una visión externa del problema, en este caso se inserta en la realidad de los trabajadores y asume un rol consultor, guía y tutor de un proceso, traspasando el conocimiento y la labor operativa a los miembros del Grupo Ergo.

Por su parte el Grupo Ergo posee un conocimiento acabado de los problemas de los trabajadores, así como el detalle de los procesos, puestos de trabajo, tareas que se ejecutan que tienen relación con la generación de TME. El Grupo Ergo aplicará las herramientas de evaluación previa capacitación por parte del Ergónomo en las tareas seleccionadas en conjunto, para luego ser parte activa de la propuesta de medidas de control.

Previo a la ejecución de las etapas del PEP, se debe comprometer a la Dirección de la empresa. Para esto, el Ergónomo asesor elaborará una breve presentación en la cual explicará la problemática actual y en qué consiste el programa de Ergonomía Participativa. EL PEP consta de 5 etapas; la propuesta inicial es el cumplimiento

de estas etapas en un período que no supere los 6 meses de trabajo (esto sólo considerando la implementación de medidas de control simples). Este período podría variar según la realidad de la empresa; es posible que por razones operativas o de producción estos plazos no se cumplan exactamente, sin embargo, no es conveniente alargar esta situación indefinidamente.

\section{Etapas del PEP}

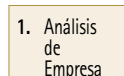

Empresa
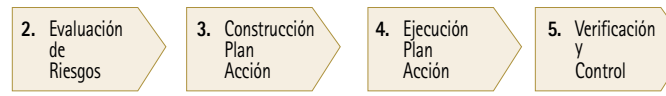

Los objetivos de las etapas del PEP se describen a continuación:

\section{Etapa 1. Análisis de Empresa}

En esta etapa se desarrollan una serie de actividades que apuntan a identificar la situación de la empresa en las temáticas de Ergonomía relacionados con la presencia de TME a partir de las cuales se define cuál es la estrategia a seguir con la empresa.

El Ergónomo iniciará la etapa de diagnóstico en la empresa, mediante consultas que permitan identificar los sectores o líneas de proceso con tareas y/o puesto de trabajo con problemas musculoesqueléticos. Se deben obtener datos respecto a la accidentabilidad de la empresa, enfermedades profesionales reportadas y sintomas musculoesqueléticos (según reporte interno de las empresas).

\section{Etapa 2. Evaluación de riesgos}

La etapa de evaluación de riesgos busca identificar los peligros y evaluar los riesgos de los distintos procesos, áreas y puestos de trabajo de la empresa. Esta etapa contempla la conformación y capacitación de un grupo de Ergonomía (Grupo Ergo) para que ejecute estas tareas.

En esta etapa se encuentran las acciones que demandan la mayor cantidad de actividades por parte del Ergónomo asesor, ya que contempla la selección de tareas, la capacitación del Grupo Ergo y la aplicación de las metodologías de evaluación. Estas metodologías son:

- Método Manual Assements Charts 12 para tareas de levantamiento-descenso y transporte de carga. ${ }^{13}$

- Tablas de Liberty Mutual para tareas de empuje y arrastre de carga. $^{14}$

\section{- Lista de Chequeo TMERT MINSAL. ${ }^{15}$}

\section{Etapa 3. Construcción plan de acción}

En esta etapa se elabora el plan que contiene las medidas de control a implementar. Contempla una reunión de análisis en la cual el Ergónomo se reúne con representantes de las diferentes áreas y se acuerdan las medidas simples a efectuar. El plan contiene medidas (simples o complejas), plazos y responsables de estas actividades. Esta investigación pretende abordar fundamentalmente las medidas de nivel simple .

\section{Etapa 4. Ejecución plan de acción}

En esta etapa se ejecutan las actividades asociadas a las medidas de control propuestas y aceptadas por la empresa, según plan de acción.

El Grupo Ergo, con apoyo del Ergónomo monitoreará que se cumpla la implementación de medidas propuestas en el Plan de acción, involucrando a la línea de supervisión y jefaturas intermedias. Se estima que un plazo acorde adecuado para la implementación de medidas simples no debiera superar los tres meses. 


\section{Etapa 5. Verificación y control}

En esta etapa se controla la ejecución de las actividades planificadas y acordadas con la empresa y se verifica la efectividad de ellas en relación a la reducción del nivel de riesgo.

\section{Universo del Estudio}

Se seleccionó una empresa manufacturera del sector industrial que posee procesos de fabricación de elementos de fijación (pernos, tornillos) y envases industriales de metal y plástico. Estos 3 sectores se identifican como: AS (elaboración de elementos de fijación), Metales (elaboración de tambores metálicos industriales) y Plástico (elaboración de envases plásticos).

El criterio utilizado fue abordar al menos 3 puestos de trabajo por Sector, que representen la ejecución de tareas críticas y que tengan representación en los integrantes del Grupo Ergo. De esta manera se focaliza el ámbito de la aplicación del programa y se consigue representar a los diferentes sectores de la empresa.

Los puestos de trabajo seleccionados representan un total de 18 tareas, de las cuales 10 de ellas corresponden a tareas de naturaleza repetitiva y 8 a tareas de manipulación de cargas.

\section{RESULTADOS}

Los resultados se presentan a continuación respecto a la aplicación del PEP en una empresa industrial. Se plantean según las etapas planteadas en la metodología, dando énfasis a la evaluación de los riesgos por parte del Grupo Ergo y en la implementación y verificación de medidas de control.

\section{Etapa 1. Análisis de Empresa}

Según tipo de accidente, el dato de relevante para este programa es el que se relaciona con la generación de TME. Para ello tomaremos como referencia el ítem "Sobre esfuerzo por peso excesivo o movimiento brusco". Analizando la casuística de accidentes entre el período enero 2013 a diciembre 2013, sólo se han reportado 8 casos en la mutualidad, lo que ha generado un total de 43 días perdidos entre ambas empresas. La Tabla $\mathrm{N}^{\circ} 1$ ilustra esta situación.

Las estadísticas ilustran que los ingresos por Sobre esfuerzo por peso excesivo o movimiento brusco, asociado a la generación de TME se encuentra en el cuarto lugar de importancia respecto al

\section{Tabla 1.}

Casuística de accidentes de la empresa entre el período de enero 2013 a diciembre de 2013.

$\begin{array}{lcc}\text { TIPO DE ACCIDENTE } & \text { No casos } & \text { No días perdidos } \\ \text { Caída del mismo nivel } & 4 & 16 \\ \text { Caída de distinto nivel } & 0 & 0 \\ \text { Golpeado por/con/contra objetos materiales o estructuras } & 25 & 372 \\ \text { Atrapamiento en máquinas, materiales o instalación } & 2 & 26 \\ \text { Contacto con objetos cortantes } & 16 & 239 \\ \text { Contacto con objetos punzantes } & 1 & 6 \\ \text { Contacto con fuego u objetos calientes } & 0 & 0 \\ \text { Proyección de partículas } & 4 & 50 \\ \text { Proyección de liquidos } & 2 & 2 \\ \text { Exposición al arco eléctrico } & 0 & 0 \\ \text { Sobre esfuerzo por peso excesivo o movimiento brusco } & 8 & 43 \\ \text { Atropellamiento o choque } & 0 & 0 \\ \text { Otros tipos } & 12 & 35 \\ \text { Total } & 50 & 789\end{array}$

número de casos reportados y representa sólo un 5,4\% del total de días perdidos de las empresas involucradas.

Es importante señalar que pese a que la notificación de casos a la mutualidad es baja, esto no representa la relevancia de este tema al interior de la empresa. La estadística precedente sólo representa los casos notificados y acogidos por la mutualidad; sin embargo, no considera a los trabajadores que refieren sintomas musculoesqueléticos y que son atendidos en el policlínico de la empresa. Los datos entregados por la empresa indican que entre un período comprendido entre enero a septiembre de 2013 se han reportado 242 consultas por dolencias musculoesqueléticas, de las cuales 135 son dolores inespecíficos (algias musculares, articulares), 92 se presentan en la zona lumbar y 15 se localizan en la extremidad superior.

\section{Etapa 2. Evaluación de riesgos \\ Conformación Grupo Ergo}

El Grupo de Ergonomía de la empresa (Grupo Ergo) quedó conformado por 9 trabajadores. El detalle de los cargos y áreas al cual representan se describe a continuación:

- 2 Prevencionistas de riesgo: 1 representante del sector metales y 1 representante de plástico y A.S.

- 3 Supervisores de área.

- 2 trabajadores representantes de tareas críticas (prensista y tornero).

- 2 Encargados de mantención de la empresa.

\section{Capacitación}

El Ergónomo capacitó a este Grupo en la aplicación en 2 sesiones de 4 horas cada una. En la primera sesión se realizó la Capacitación en la aplicación de la Norma Técnica MINSAL TMERT. ${ }^{16}$ Los contenidos generales tratados fueron los siguientes.

- Introducción a Trastornos Musculoesqueléticos en el trabajo.

- Factores de riesgo: repetitividad, postura, fuerza, tiempos de recuperación, factores adicionales.

- Aplicación de Lista de Chequeo MINSAL.

- Taller práctico: operador de máquina rebabadora.

Luego, se entregó el Software ACHS Ergobrazos 3.0 y se designaron del grupo Ergo a los responsables de realizar las evaluaciones de las tareas repetitivas seleccionadas por empresa, área y sector.

La segunda sesión de capacitación se realizó en dos semanas posteriores con el fin de que en ese período de tiempo el Grupo Ergo evaluara las tareas repetitivas indicadas. Esta sesión se trató sobre los métodos de evaluación de manejo manual de carga exigida por la ley 20.001. Los contenidos generales tratados fueron los siguientes:

- Conceptos de Manejo Manual de Carga.

- Ley 20.001 y Decreto Supremo N63.

- Método MAC: aplicación.

- Tablas de Liberty Mutual: aplicación.

- Taller práctico: se resuelve ejemplo solicitado al Grupo Ergo. Siguiendo la metodología anterior se resuelve entregar tareas y responsables al Grupo Ergo, de la misma manera se entregó el Software ACHS Ergocargas 2.0 como apoyo para la resolución de casos. Por último, se acordó con el Grupo Ergo que las evaluaciones de empuje y arrastre serían realizadas por el Ergónomo, ya que se consideraron de una mayor complejidad.

Aplicación de metodologías

Durante un período de 3 semanas el Grupo Ergo aplicó la Lista de Chequeo MINSAL y Método MAC a las tareas seleccionadas. 
Durante este proceso, la acción del Ergónomo fue de consulta, aprovechando las instancias de visita a la empresa para resolver dudas y verificar la correcta aplicación.

Como se mencionó anteriormente, las tareas de empuje-arrastre y aquellas que revestían una mayor complejidad fueron evaluadas por el Ergónomo. El valor de fuerza resultante se obtuvo mediante un dinamómetro digital (marca Mecmesin AFG 2.5N). Los resultados obtenidos se compararon con las Tablas de Liberty Mutual de empuje y arrastre utilizando como criterio de fuerza "aceptable" aquel que protege al 75\% de la población. ${ }^{14}$

Existen dos tareas (levantamiento parcial de tambores en zona de pintura y molino) que por su particular ejecución se deben evaluar con criterios específicos establecidos en la Norma UNE-EN 10053:2002 (Tabla 1: fuerza isométrica FB ámbito profesional en Newton).

\section{Evaluación de tareas repetitivas}

Los resultados de la aplicación de la Lista de Chequeo TMERT MINSAL por parte del Grupo Ergo se presentan por sector y tarea evaluada en las tablas № 2, 3 y 4.

Tabla 2.

Resultados de aplicación Lista de Chequeo MINSAL TMERT en Sector Metales.

\begin{tabular}{|c|c|c|c|c|c|c|}
\hline \multirow[t]{2}{*}{ Sector } & \multirow[t]{2}{*}{ Tarea } & \multirow{2}{*}{$\begin{array}{l}\text { Trabajadores } \\
\text { por puesto }\end{array}$} & \multicolumn{4}{|c|}{ NIVEL DE RIESGO } \\
\hline & & & Repetitividad & Postura & Fuerza & $\begin{array}{l}\text { Tiempos de } \\
\text { recuperación } \\
\text { o descanso }\end{array}$ \\
\hline Metales & $\begin{array}{l}\text { Alimentación } \\
\text { tapas línea } \\
\text { Merko }\end{array}$ & 2 & & & & \\
\hline Metales & Perforado & 1 & & & & \\
\hline Metales & Inserción & 2 & & & & \\
\hline
\end{tabular}

Como se puede observar, en todas las tareas evaluadas se verifica la presencia de repetitividad y postura, las cuales son calificadas con riesgo "rojo". El factor repetitividad se explica por la presencia de ciclos de trabajo cortos (por debajo de los 30 segundos) y tiempo de exposición superior a las 4 horas totales en la jornada de trabajo, según datos de la producción. El factor postura se explica por la presencia de posturas forzadas de las extremidades superiores durante la ejecución de la tarea (principalmente segmento muñeca para alimentación de tapas y hombro para inserción y perforado).

El factor fuerza fue evaluado con color "verde" debido a que fue considerado con valor inferior a 3 puntos según la escala de Borg. Es decir, la fuerza percibida por los trabajadores es débil en relación a tomar las tapas y posicionarlas en las máquinas correspondientes.

En relación a los tiempos de recuperación, este fue calificado con color "rojo" para la tarea de inserción y perforado; dado que no se presentan pausas formales, los tiempos de recuperación son insuficientes y se trabaja la mayor parte del tiempo por más de una hora consecutiva sin pausa o variación de la tarea.

Respecto a los factores adicionales y organizacionales/psicosociales se identifica en todas las tareas la presencia de temperaturas frías en período de invierno (según percepción de los trabajadores) y un ritmo impuesto por otras personas asociado a la producción. Como se puede observar, en todas las tareas evaluadas se verifica la presencia de repetitividad y postura, las cuales son calificadas con riesgo "rojo". Esto se explica por el tiempo de exposición superior a las 4 horas totales en la jornada de trabajo, ciclos de
Tabla 3.

Resultados de aplicación Lista de Chequeo MINSAL TMERT en Sector Plástico.

\begin{tabular}{|c|c|c|c|c|c|c|}
\hline \multirow[t]{2}{*}{ Sección } & \multirow[t]{2}{*}{ Tarea } & \multirow{2}{*}{$\begin{array}{c}\text { Trabajadores } \\
\text { por puesto }\end{array}$} & \multicolumn{4}{|c|}{ NIVEL DE RIESGO } \\
\hline & & & Repetitividad & Postura & Fuerza & $\begin{array}{l}\text { Tiempos de } \\
\text { recuperación } \\
\text { o descanso }\end{array}$ \\
\hline Decorado & $\begin{array}{l}\text { Instalación } \\
\text { asas } \\
\text { Kase } 2\end{array}$ & 2 & & & & \\
\hline Decorado & $\begin{array}{l}\text { Alimentación } \\
\text { baldes a } \\
\text { máquina Moss } 3\end{array}$ & 3 & & & & \\
\hline Decorado & $\begin{array}{l}\text { Instalación de } \\
\text { asas plásticas }\end{array}$ & 3 & & & & \\
\hline
\end{tabular}

trabajo cortos (por debajo de los 30 segundos) y la presencia de posturas forzadas de las extremidades superiores durante la ejecución de la tarea principalmente a nivel de muñeca y codo.

Por otra parte, en las tareas de Instalado de asas en la máquina Kase 2 y de inserción de baldes a la máquina Moss 3, se calificó el Tiempo de recuperación y descanso como "rojo", lo que indica que no se identificaron pausas ni tiempos de recuperación adecuados (considerando la peor condición los trabajadores trabajaban de manera continua 1 hora sin pausa o tiempo de recuperación dentro del ciclo).

El factor fuerza fue considerado con valor 3 según la escala de Borg (moderado). Es decir, la fuerza percibida por los trabajadores es moderada y se califica en color amarillo según lo indica la Norma Técnica MINSAL. Este factor es variable según la técnica utilizada, generalmente los trabajadores nuevos sin experiencia tienen una mayor percepción del esfuerzo realizado.

Por último, respecto a los factores adicionales y organizacionales/ psicosociales se identifica en todas las tareas de inserción de asas un trabajo que requiere precisión y uso simultáneo de fuerza (según la técnica utilizada) además de un ritmo impuesto por otras personas asociado a la producción para todas las tareas.

Tabla 4.

Resultados de aplicación Lista de Chequeo MINSAL TMERT en A. S.

\begin{tabular}{llc|c|c|c} 
Sección & Tarea & $\begin{array}{c}\text { Trabajadores } \\
\text { por puesto }\end{array}$ & $\begin{array}{c}\text { NIVEL DE RIESGO } \\
\text { Repetitividad Postura }\end{array}$ & $\begin{array}{r}\text { Fuerza } \\
\text { Tiempos de } \\
\text { recuperación } \\
\text { o descanso }\end{array}$ \\
\hline Forjado & Terrajado & 3 & & & \\
\hline Forjado & $\begin{array}{l}\text { Rebabado } \\
\text { pieza metálicas }\end{array}$ & 3 & & & \\
\hline Forjado & Esmerilado & 2 & & & \\
\hline Forjado & $\begin{array}{l}\text { Punteado } \\
\text { de pernos }\end{array}$ & 3 & & & \\
\hline
\end{tabular}

Como se puede observar, en todas las tareas evaluadas se verifica la presencia de repetitividad y postura, las cuales son calificadas con riesgo "rojo", a excepción de la tarea de Terrajado calificada con riesgo "amarillo". Lo anterior se explica por el tiempo de exposición superior a las 4 horas totales en la jornada de trabajo (a excepción de Terrajado), ciclos de trabajo cortos (por debajo de los 30 segundos) y la presencia de posturas forzadas de las extremidades superiores, particularmente el segmento hombro en la máquina rebabadora, esmerilado y terrajado.

El factor fuerza es considerado crítico en las tareas de Terrajado y Esmerilado; esto se explica por los esfuerzos requeridos en el accionamiento de las mordazas en la tarea de Terrajado y en el uso 
continuo y sostén del esmeril en el Esmerilado. En ambas tareas este esfuerzo fue calificado como 5 (Fuerte) según la escala de Borg.

El factor "tiempos de recuperación o descanso" se observa que el operador de la máquina rebabadora no cuenta con pausas ni tiempos de recuperación adecuados para la tarea. En el resto de las tareas este tiempo de recuperación se encuentra implícito dentro del ciclo de trabajo.

Por último, respecto a los factores adicionales y organizacionales/ psicosociales en la tarea de esmerilado, se presenta un número importante de factores tales como: uso frecuente o continuo de herramientas vibrantes, exposición a frío en invierno y calor en verano, ya que el puesto se encuentra fuera de la planta, el equipamiento de protección personal restringe la habilidad del trabajador, se sostienen herramientas de manera continua, se realizan fuerzas de manera estática y el trabajador realiza su tarea aislado del proceso de producción.

Por otra parte, se destaca en la tarea de rebabado de piezas el uso de o manipulación de herramientas de manera continua (pinzas tipo tenazas para tomar los pernos) y el ritmo de trabajo impuesto por la producción.

\section{Evaluación de tareas de manipulación de cargas}

La Tabla No5 resume las tareas de empuje y arrastre evaluadas por Ergónomo; luego, la Tabla Nº6 resume los resultado de la aplicación del método MAC por Grupo Ergo.

Como se puede observar, todas las tareas evaluadas presentan niveles no tolerables de riesgo.

Tabla 5.

Resumen de tareas de empuje y arrastre utilizando dinamómetro digital.

\begin{tabular}{|c|c|c|c|c|c|}
\hline $\begin{array}{l}\text { Tarea } \\
\text { (peso kg) }\end{array}$ & Frecuencia & $\begin{array}{l}\text { Distancia } \\
\text { (metros) }\end{array}$ & $\begin{array}{l}\text { Valor } \\
\text { medido } \\
(\mathrm{Kg}-\mathrm{f})\end{array}$ & $\begin{array}{l}\text { Límite } \\
\text { permisible fuerza } \\
\text { inicial }(\mathrm{Kg}-\mathrm{f})\end{array}$ & Riesgo \\
\hline $\begin{array}{l}\text { Levante parcial } \\
\text { tambor con } \\
\text { molido }(110 \mathrm{~kg})\end{array}$ & $\begin{array}{l}60 \text { veces } \\
\text { al dia }\end{array}$ & - & $28,8 \mathrm{~kg}-\mathrm{f}$ & $\begin{array}{l}14,5 \mathrm{~kg}-\mathrm{f} \\
\text { según Norma } \\
\text { UNE-EN 1005-3 }\end{array}$ & $\begin{array}{l}\text { No } \\
\text { tolerable }\end{array}$ \\
\hline $\begin{array}{l}\text { Empuje carro } \\
\text { con tapas } \\
(904 \mathrm{~kg})\end{array}$ & $\begin{array}{l}6 \text { veces } \\
\text { al dia }\end{array}$ & $2 m$ & $47,4 \mathrm{~kg}-\mathrm{f}$ & $\begin{array}{l}34 \mathrm{~kg}-\mathrm{f} \text { según } \\
\text { Tablas de Liberty } \\
\text { Mutual }\end{array}$ & $\begin{array}{l}\text { No } \\
\text { tolerable }\end{array}$ \\
\hline $\begin{array}{l}\text { Arrastre traspaleta } \\
\text { con tambores de } \\
\text { pintura llenos } \\
\text { (700 kg aprox.) }\end{array}$ & $\begin{array}{l}8 \text { veces } \\
\text { al dia }\end{array}$ & $20 \mathrm{~m}$ & $40 \mathrm{~kg}-\mathrm{f}$ & $\begin{array}{l}26 \mathrm{~kg}-\mathrm{f} \text { según } \\
\text { Tablas de Liberty } \\
\text { Mutual }\end{array}$ & $\begin{array}{l}\text { No } \\
\text { tolerable }\end{array}$ \\
\hline $\begin{array}{l}\text { Levante parcial } \\
\text { tambor con pintura } \\
\text { (180 kg aprox.) }\end{array}$ & $\begin{array}{c}\text { 3-4 veces } \\
\text { al día }\end{array}$ & - & $95 \mathrm{~kg}-\mathrm{f}$ & $\begin{array}{l}14,5 \mathrm{~kg}-\mathrm{f} \\
\text { según Norma } \\
\text { UNE-EN 1005-3 }\end{array}$ & $\begin{array}{l}\text { No } \\
\text { tolerable }\end{array}$ \\
\hline
\end{tabular}

Tabla 6.

Resumen de tareas evaluadas con Método MAC Grupo Ergo.

\begin{tabular}{lll|l} 
Sector & Puesto Involucrados & Actividad / Tarea & \multicolumn{2}{l}{ ÍNDICE DE RIESGO } \\
L/D T EA LD/e
\end{tabular}

L/D: Levantamiento/Descenso. T: Transporte. EA: Empuje y Arrastre.

LD/e: Levantamiento y descenso en equipo.

Como se puede observar, 3 de las 4 tareas evaluadas presentan niveles de riesgo no tolerables (en una escala de Pinder de 4 niveles, estas tareas se encuentran en un nivel de riesgo 3).

\section{Etapa 3. Construcción plan de acción}

Se utilizó una estrategia en la que el Ergónomo tiene un rol de moderador y guía, entregando el espacio necesario para el desa- rrollo de ideas que apunten a la solución del problema. En primera instancia se analizó el diagnóstico realizado, las tareas con riesgo crítico y particularmente los factores de riesgo que fueron calificados con color "rojo"; luego se propusieron medidas para el control de dichos factores.

Como material de apoyo para construir el plan de acción se utilizó la Guía Técnica de Manejo Manual de $\operatorname{Carga}^{12}$ y la Guía Técnica de Prevención de TME. ${ }^{17}$ Es importante destacar que la mayor parte de las soluciones propuestas nace de los mismos trabajadores, quien en su mayoría ya tenía previamente identificado como contribuir a la solución del problema.

Las medidas que se presentarán a continuación fueron acordadas con el Grupo Ergo. Existen medidas Ingenieriles y administrativas que buscan eliminar o reducir el riesgo de generar TME en las tareas evaluadas. El plan de acción contempla el tipo de medida: simple o compleja de implementar y el o los responsables directos de implementarla.

Como acuerdo se establece que las medidas simples -tales como pausas, rotaciones de puesto de trabajo- se implementen en un plazo no superior a 3 meses según criterio establecido en la Norma Técnica MINSAL TMERT, mientras que las medidas más complejas (ej. automatización de máquinas o parte de ellas) se analizará con más detalle mediante la asesoría del Ergónomo.

\section{Etapa 4 y 5. Implementación de medidas de control, verificación y control}

Se acordó que la implementación de medidas de control estará liderada en el interior de la empresa por 2 representantes del Grupo Ergo que, en este caso, serán los Prevencionistas de riesgos que se encuentran a cargo de los distintos sectores. Luego, se establecieron reuniones periódicas durante las cuales se realizaron visitas inspectivas a las diferentes áreas, sectores y puestos de trabajo en los que se propusieron medidas de control. Se acordó que el tiempo máximo de la implementación de medidas simples sería de 3 meses.

El resumen de las medidas de control propuestas y su nivel de cumplimiento se muestran en la Tabla $\mathrm{N}^{\circ} 7$ para medidas de nivel simple y la Tabla No8 para medidas de nivel complejo. Luego se muestran ejemplos de medidas de carácter ingenieril aplicadas.

Sobre un total de 14 medidas simples, se observó en terreno el cumplimiento de 11 de ellas, lo que equivale a un 79\% de cumplimiento. El resultado de la implementación de estas medidas implica una reducción del nivel de riesgo a niveles tolerables (amarillo-verde) en las tareas de naturaleza repetitiva.

Por otra parte existen 3 medidas no implementadas que corresponden a las tareas de rebabado, esmerilado y punteado de pernos. Sobre un total de 15 medidas simples, se observó en terreno el cumplimiento de 4 de ellas, lo que equivale a un $27 \%$ de cumpli-
Figura 1.

Mesa con polines para el deslizamiento de tambores.

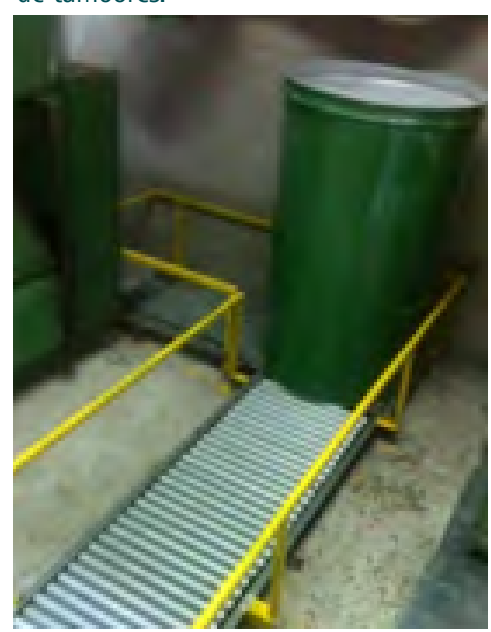


Tabla 7.

Medidas de control simples según tarea y sector.

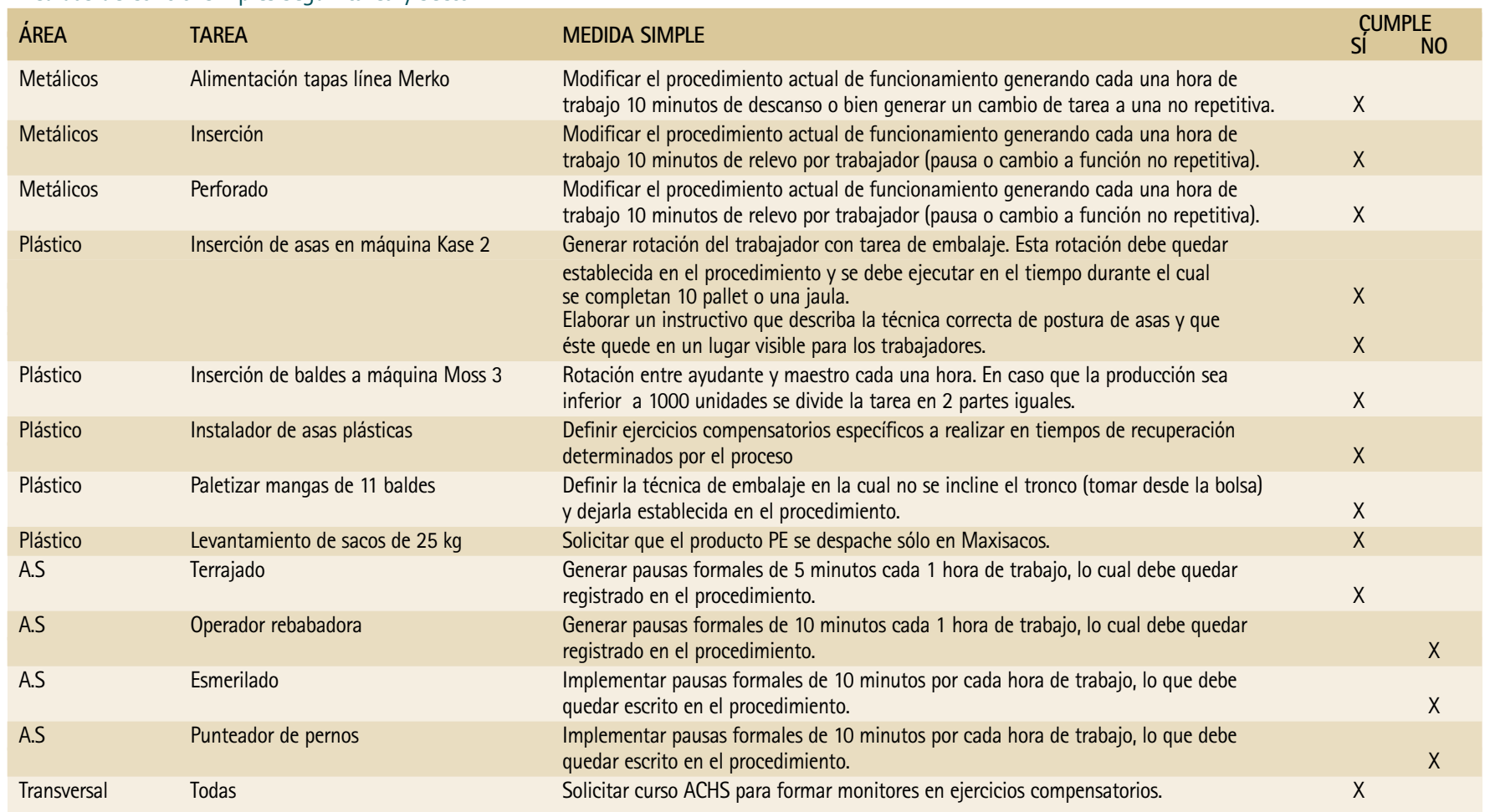

Tabla 8.

Medidas de control complejas según tarea y sector

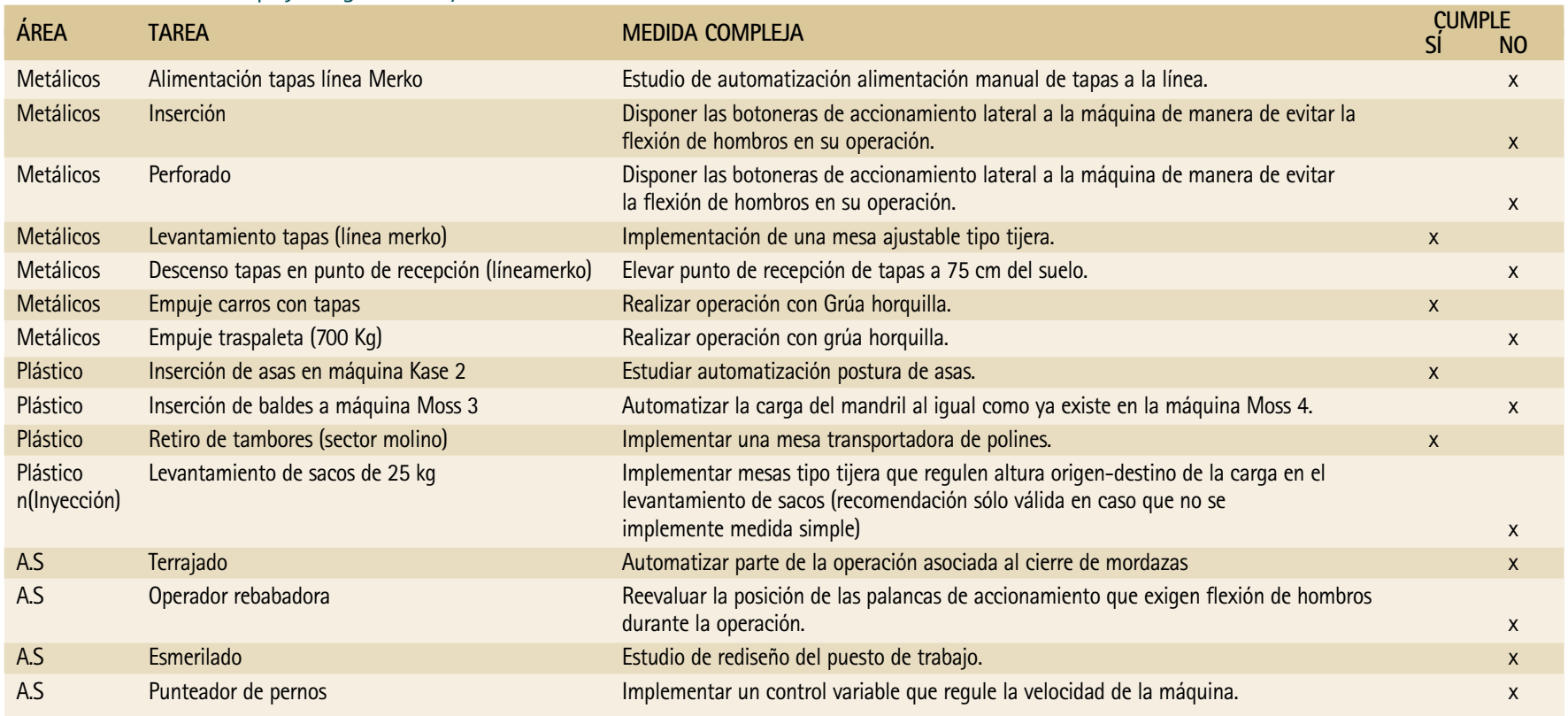

miento. El resultado de la implementación de estas medidas implica una reducción del nivel de riesgo a nivel tolerable para la tarea de levantamiento de tapas en la línea merko y la eliminación del riesgo para las tareas de empuje de carros con tapas, retiro de tambores e Inserción de asas en máquina Kase 2.

Por otra parte, existen 11 medidas no implementadas. Se pudo verificar que la mayor parte de ellas se encuentra en proceso de evaluación y cotización con proveedores.
A modo de ejemplo se ilustra una de las medidas complejas implementadas en sector plástico (molino); con la finalidad de disminuir la fuerza requerida para movilizar los tambores, la empresa con recursos propios fabricó una mesa con polines para instalarla en el sitio de recolección de plástico molido. De esta manera se elimina la acción de levantar parcialmente el tambor y transportarlo en forma manual mediante giros. La Figura 1 muestra la fabricación de este accesorio. 


\section{ANÁLISIS Y CONCLUSIONES}

La experiencia de Ergonomía participativa aplicado a empresas ha sido publicado en países como Canadá, USA, Finlandia y Australia con buenos resultados en cuanto al impacto positivo en sintomas musculoesqueléticos, reducción de lesiones, compensaciones laborales y reducción de los días perdidos. Sin embargo, la magnitud de estos efectos requiere una definición más precisa. ${ }^{2}$

Por otra parte, países como España han publicado metodologías de Ergonomía Participativa aplicado a su realidad legal con buenos resultados incipientes. ${ }^{18}$

El presente proyecto, con las limitaciones propias del estudio, constituye uno de los pioneros en Chile de aplicación de un programa de Ergonomía Participativa en forma sistemática en un período breve de tiempo. Los resultados preliminares son alentadores respecto a la implementación de medidas de control, compromiso de los trabajadores y compromiso de los ejecutivos de la empresa.

Otro de los puntos a destacar es que este tipo de programa es adaptable a las condiciones propias de la empresa. Sin embargo, hay que decir que la propia participación no está exenta de dificultades. En primer lugar, es necesario motivar para la participación, no todo el mundo está dispuesto a participar, sobre todo si no cuenta con la información y las garantías suficientes de que ello puede resultar beneficioso. También hay que prestar atención a que determinados sectores en la empresa no se sientan excluidos, lo que podría generar malestar, dificultar o impedir la colaboración para alcanzar el éxito del proyecto. Por último, es importante no provocar expectativas que no puedan cumplirse, porque generan frustración y desmotivación. , $^{78}$

Sobre el punto anterior, vale la pena recoger la opinión del Grupo Ergo, el que ha manifestado, dentro de las dificultades propias del proceso, problemas de comunicación y, en ocasiones, falta de reconocimiento de sus pares respecto a la función asignada, lo que desmotiva su rol preventivo en la empresa.

Sobre los aspectos a destacar, el Grupo Ergo manifiesta satisfacción de haber conocido la disciplina de la Ergonomía, poder aplicarla en su labor diaria, proponer medidas de control y luego verlas implementadas. Asimismo, mencionan la generación de un lenguaje Ergonómico al interior de la empresa, lo que facilita a futuro mejoras en el ámbito global de lo que implica una visión amplia de esta disciplina.

Estudios respecto a la evaluación económica de un programa de ergonomía participativa en una planta textil señalan que este tipo de intervenciones pueden ser una buena relación costo-beneficio para las proyecciones de la empresa. ${ }^{19} \mathrm{Al}$ analizar este punto se pudo apreciar que en el presente proyecto la empresa asumió costos razonables en la implementación de medidas complejas, siendo la mayor parte de estas absorbidas por los costos fijos asociados a las áreas de mantención propia. En lo que refiere a inversión, la empresa no incurrió en gastos extraordinarios, más aun considerando que el PEP fue ofrecido como parte de la asesoria brindada por la Mutualidad (Ley 16.744), lo cual no tiene un costo adicional.

$\mathrm{Al}$ comparar el PEP con las asesorias estándares brindadas por las mutualidades en el ámbito de la Ergonomía, se puede deducir de este proyecto que el PEP logra ventajas comparativas altamente favorables ya que contribuye a comprometer a la empresa en mejorar su cultura preventiva, establece una relación directa con los involucrados, consigue asesorar a la empresa en el cumplimiento normativo y logra en el tiempo un nivel de implementación de medidas de control superior a lo esperado.

Si bien el tiempo dedicado a la empresa es mayor que una asesoría técnica puntual, se debe señalar que el trabajo operativo del Ergónomo se traspasa en conocimiento al Grupo Ergo, quien ejecuta el levantamiento de tareas, aplica las herramientas de evaluación y propone gran parte de las medidas de control. Según esta visión, el Ergónomo debe ser lo suficientemente competente para lograr este objetivo y de esta manera establecer con la empresa un rol consultor y menos operativo.

Respecto a los resultados formales de este proyecto según los objetivos planteados, se concluye lo siguiente:

- Se consigue formar y capacitar un grupo de Ergonomía en la empresa asesorada según los parámetros establecidos en la literatura técnica.

- Se logró que los integrantes del Grupo Ergo aplicaran las herramientas de evaluación contendidas en la normativa nacional previo a una capacitación formal y dirigida a los puestos de trabajo seleccionados.

- Se consigue que la empresa en conjunto con la mutualidad definan un plan de acción con medidas de control simples y complejas bien enfocadas y dirigidas a la solución del problema diagnosticado.

- Se consigue implementar un 79\% de las medidas simples acordadas y además un $27 \%$ de las medidas complejas, con lo cual se superan las expectativas respecto al objetivo inicial propuesto.

- Por último, se consigue cumplir con la legislación nacional en temas de Ergonomía, ya que lo que solicitan las autoridades a las empresas son programas de prevención de TME según lo indicado en la Norma Técnica TMERT MINSAL y la Guía Técnica de Manejo Manual de Carga. Lo anterior es uno de los focos del PEP.

En razón a los resultados y pese a que estos son preliminares, se concluye que el PEP propuesto en este proyecto puede ser una buena estrategia a implementar en empresas que cuenten con un compromiso real por parte de la gerencia, un grupo de trabajadores motivados en solucionar problemas de Ergonomía y un Ergónomo competente que guíe y dirija el proceso. 


\section{REFERENCIAS}

1. Instituto de Salud Pública de Chile. Guía para la gestión del riesgo asociado al manejo manual de carga (MMC); Primera versión [en línea]. Santiago de Chile: MINSAL; 2013 [citado mar 2015]. Disponible en: http://www.sochergo.cl/ docs/1395323950 guia para la gestion del riesgo asociado al manejo manual de carga primera version 2013.pdf

2. Rivilis I, Van Eerd D, Cullen $K$, Cole D, Irvin E, Tyson J, Mahood Q. Effectiveness of participatory ergonomic interventions on health outcomes: a systematic review. Appl ergon. 2008;39:342-358

3. García A. Análisis de las enfermedades profesionales de la achs: descripción de la situación en el periodo 1995-2009. Cienc Trab. 2011;13(40):107-112.

4. Asociación Chilena de Seguridad (ACHS). Gerencia de prevención. Base de datos estadisticas; documento interno. Santiago de Chile: ACHS [s.p.]; 2012.

5. Haines $\mathrm{H}$, Wilson JR. Development of a framework for participatory ergonomics. London: HSE Books; 1998. Health and Safety Executive Contract Research Report; 174.

6. Cole D, Rivilis I, Van Eerd D, Cullen K, Irvin E, Kramer D. Effectiveness of participatory ergonomic interventions: a systematic review. Toronto: Institute for Work \& Health; 2005.

7. Gadea R, Sevilla MJ, Garcia AM. Ergopar; Un procedimiento de ergonomía participativa para la prevención de trastornos musculoequeléticos de origen laboral. Madrid: Instituto Sindical de Trabajo, Ambiente y Salud (ISTAS); 2009.

8. Gjessing C, SchoenbornT, Cohen A. Participatory ergonomic interventions in meatpacking plants. Atlanta: CDC; 1994. NIOSH Publication; 124.

9. Estill $C$, Steege $A$, lalich N. Simple solutions: ergonomics for farm workers. Atlanta: CDC; 2001. NIOSH Publication; 111.

10. Agencia Europea para la Seguridad y la Salud en el Trabajo (AESST). Introducción a los trastornos musculoesqueléticos de origen laboral. Madrid: OSHA; 2007. Factsheet; 71

11. Asociación Chilena de Seguridad (ACHS). Gerencia de prevención. Sistema preventivo 2010; documento interno. Santiago de Chile: ACHS [s.p.]; 2011.

12. Monnington $S$, Quarrie $C$, Pinder $A$, Morris I. Development of manual handling assessment charts (MAC) for health and safety inspectors. In: Contemporary Ergonomics. London: Taylor \& Francis; 2003.

13. Chile. Ministerio del Trabajo. Guía técnica para la evaluación y control de los riesgos asociados al manejo o manipulación manual de carga [en línea]. Santiago de Chile: MINTRAB; 2008 [citado Mar 2015]. Disponible en: http:// www.dt.gob.cl/1601/simple-article-95553.html
14. Snook SH, Ciriello VM. The design of manual handling tasks: revised tables of maximum acceptable weights and forces. Ergonomics. 1991;34(9):1197-1213.

15. ISO 11228-3:2007. Ergonomics; Manual handling .Part 3: handling of low loads at high frequency [on line]. Geneva: ISO; 2010 [cited Mar 2015]. Available on: https://www.iso.org/obp/ui/\#iso:std:iso:11228:-3:ed-1:v2:en

16. Chile. Ministerio de Salud. Norma técnica de identificación y evaluación de factores de riesgo de trastornos musculoesqueléticos relacionados al trabajo. Santiago de Chile: MINSAL; 2011.

17. Asociación Chilena de Seguridad (ACHS). Gerencia de prevención. Guia técnica para la prevención de riesgos asociados a trastornos musculoesqueléticos de extremidades superiores. Santiago de Chile: ACHS [s.p.]; 2011.

18. Garcia AM, Gadea R, Sevilla MJ, Genis S, Ronda E. Ergonomía participativa: empoderamiento de los trabajadores para la prevención de trastornos musculoequeléticos. Rev Esp Salud Pública. 2009 83:509-518.

19. Tompa $E$, Dolinschi R, Laing A. An economic evaluation of participatory ergonomics process in an auto parts manufacturer. J Safety Res. 2009;40:41-47.

\section{Referencias complementarias:}

Álvarez-Casado E, Hernández-Soto A, Tello S. Manual de evaluación de riesgos para la prevención de trastornos musculoequeléticos. Barcelona: FH; 2009.

Asociación Chilena de Seguridad (ACHS). Gerencia de prevención. Base de datos estadisticas; documento interno. Santiago de Chile: ACHS [s.p.]; 2013.

Ciriello V, Snook S. Survey of manual handling tasks. Int J Ind Ergonom. 1999; 23:149-156.

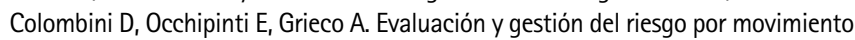
repetitivo de las extremidades superiores: análisis organizativo, indices de exposición OCRA, pautas de intervención, principios de rediseño. Santiago de Chile: ACHS; 2005. Colección Cátedra Mutual CYCLOPS UPC.

Eyquem I, Córdova V, Maldonado 0. Usabilidad, confiabilidad y validez de un método para la evaluación de factores de riesgos en el manejo manual de cargas. Cienc Trab. 2007;9(25):135-142.

Grieco A, Molteni G, de Vito G, Sias N. Epidemiology of musculoskeletal disorders due to biomechanical overload. Ergonomics. 1998;41(9):1253-1260.

Koningsveld E, Dul J, Van Rhijn G, Vink P. Enhancing the impact of ergonomics interventions. Ergonomics.2005; 48:559-580.

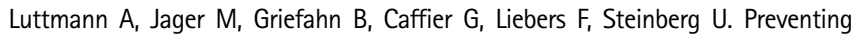
musculoskeletal disorders in the workplace. Geneva: WHO; 2003. Preventing Workers Health Series; 5. 